\title{
End-of-life care pathway of head and neck cancer patients : single-institution experience
}

\section{Heinonen, $T$.}

2018-02

Heinonen , T, Loimu , V , Saarilahti , K , Saarto , T \& Mäkitie , A 2018 , ' End-of-life care pathway of head and neck cancer patients : single-institution experience ' , European Archives of Oto-Rhino-Laryngology , vol. 275 , no. 2 , pp. 545-551 . https://doi.org/10.1007/s00405-017-4843-x

http://hdl.handle.net/10138/300069

https://doi.org/10.1007/s00405-017-4843-x

publishedVersion

Downloaded from Helda, University of Helsinki institutional repository.

This is an electronic reprint of the original article.

This reprint may differ from the original in pagination and typographic detail.

Please cite the original version. 


\title{
End-of-life care pathway of head and neck cancer patients: single- institution experience
}

\author{
T. Heinonen ${ }^{1} \cdot$ V. Loimu ${ }^{1} \cdot$ K. Saarilahti ${ }^{1} \cdot$ T. Saarto $^{1} \cdot$ A. Mäkitie $^{2,3}$ (])
}

Received: 28 September 2017 / Accepted: 8 December 2017 / Published online: 15 December 2017

(c) Springer-Verlag GmbH Germany, part of Springer Nature 2017

\begin{abstract}
Background Studies on palliative care of head and neck cancer (HNC) patients are scarce although the affected patient population is quite large.

Objective To evaluate the role of a specialised palliative-care pathway of $\mathrm{HNC}$ patients.

Patients and methods Data on all HNC patients who were treated at the Helsinki University Hospital Palliative Care Center during 1 year were retrospectively reviewed. The analysis comprised 60 patients (49 males; mean age 67 years; range 28-88). All patients had a minimum follow-up of 1 year or until death.

Results Fifty-nine (98\%) out of the 60 patients died during the follow-up period. Median survival after diagnosis was 11 months (range 3 weeks-11.9 years) and after withholding disease-specific therapies 3 months (range 0-16). Thirty-three (55\%) patients received palliative radiotherapy, 27 (45\%) had PEG tube and $17(28 \%)$ tracheostomy. Thirty-seven (66\%) patients visited an emergency department (ED) (median 1.3 visits; range $0-6)$ and $21(35 \%)$ were hospitalised at the university hospital during the palliative period. The most common severe complications were infection (also the most common reason for ED visits and hospitalisation), bleeding (four massive airway bleedings with one death), delirium and airway obstruction (one emergency tracheostomy). Twelve (35\%) out of the 34 patients who were referred to specialised home care died at home as compared with three (12\%) out of the 26 patients not supported by a specialised home-care team.

Conclusions Severe complications leading to an emergency unit visit and hospitalisation are common among HNC patients in their relatively short palliative period reflecting the need for early-integrated palliative care. Collaboration with a specialised palliative home-care team seems to increase end-of-life care at home.
\end{abstract}

Keywords Head and neck cancer $\cdot$ Palliative care $\cdot$ Terminal care

Heinonen and Loimu contributed equally.

Saarto and Mäkitie contributed equally.

A. Mäkitie

Antti.makitie@helsinki.fi

1 Comprehensive Cancer Center, University of Helsinki and Helsinki University Hospital, Helsinki, Finland

2 Department of Otorhinolaryngology, Head and Neck Surgery, University of Helsinki and Helsinki University Hospital, P.O. Box 263, FI-00029 Helsinki, Finland

3 Division of Ear, Nose and Throat Diseases, Department of Clinical Sciences, Intervention and Technology, Karolinska Institutet, Karolinska University Hospital, 17176 Stockholm, Sweden

\section{Introduction}

Each year approximately 800 head and neck cancers (HNCs) are diagnosed in Finland. In 2014, 292 of them were registered in the Helsinki University Hospital area with a referral area of 1.9 M [1]. The incidence and survival rates vary according to primary tumour site and stage of the disease [2-4]. Standard treatment for early-stage HNC is either surgery or radiotherapy. In locally advanced diseases, it consists of either definitive chemoradiotherapy, or surgical resection of the primary tumour and neck dissection combined with postoperative radiotherapy.

According to the literature, 5-14\% of all patients diagnosed with $\mathrm{HNC}$ are offered palliative care without any lifeprolonging treatments at first presentation [2-5]. Usually a decision for palliative approach is made due to advanced, either inoperable or metastatic cancer, poor overall health or 
other medical conditions that contraindicate cancer-specific treatments. Also, persistent or recurrent tumour growth may show resistance to all available treatment modalities. Overall survival of patients with non-curable $\mathrm{HNC}$ is poor, approximately less than 6 months [6, 7].

Palliative care of HNC patients with difficult symptoms, such as airway obstruction, dysphagia, bleeding, malodorous wounds and extreme cachexia, remains a challenge [8-10]. Patients may need a tracheostomy for compromised airway. Cachexia could be a paraneoplastic symptom, but also due to difficult or painful swallowing, and a PEG tube may be needed. Heavy symptom burden, changed body image and impaired physical functioning cause substantial psychosocial distress for the patients and their families [11]. Access to specialised palliative care is needed to ensure good quality of care and a dignified death. Previously published studies among patients with different types of cancer have found that early-integrated palliative care has led to improved quality of life and reduced costs [12-16].

Studies on palliative care of HNC patients are scarce despite the heavy symptom burden experienced by this patient population. Our aim was to describe the current status of palliative care of HNC patients in our university hospital region and to evaluate the specialised palliative care pathway between the Cancer Center Palliative Unit and palliative care providers in primary health care.

\section{Patients and methods}

This retrospective study consists of $60 \mathrm{HNC}$ patients in the palliative phase of their disease. The patients were treated at the Center of Palliative Care (Palliative Center) at the Comprehensive Cancer Center of the Helsinki University Hospital (HUH) during a 12-month-period between 1 May 2014 and 30 April 2015. The follow-up period for all patients was 12 months or until death. In this study, palliative phase is defined as a disease period when curative or life-prolonging treatment can no longer be offered. This covered the period from the decision to abstain from cancer-specific treatments (except palliative radiotherapy or surgery for symptom control) to death.

The patients were identified from hospital registries using the International Classification of Disease (ICD-10) coding for carcinoma of tongue, other sites in oral cavity, salivary glands, tonsils, pharynx (C01-C14), sinonasal area, and larynx (C30-C32). Data on patient demographics, tumour characteristics, and previous cancer treatments, as well as data on visits to the Palliative Center or emergency department and hospitalisation periods were recorded from the respective hospital charts. Data on the cause and place of death were collected from death certifications.
An institutional research approval was granted for the study design. Permission to access Finnish Cancer Registry data was granted by the National Institute of Health and Welfare.

\section{Palliative pathway}

In Finland, treatment of HNC patients is centralised to the five university hospitals. At our university hospital district, a multi-disciplinary tumour board, including surgeons, medical and radiation oncologists, radiologists, pathologists, palliative-care specialist, and a specialist nurse, is responsible for all primary treatment recommendations.

The palliative pathway of HNC patients in our hospital district was re-organised in March 2014. Since then, all patients with non-curable HNC have been systematically referred to the Palliative Center for specialist-level palliative care. Palliative Center is also responsible for coordination of the care according to a regional palliative-care pathway. The end-of-life care could be provided by a specialised hospice, specialised community hospital wards, general community hospital wards, and home care by specialised or general teams depending on the region. During the data collection time, there were specialised palliative-care services available mainly in larger cities in the HUH region. These included one hospice, two specialised wards and several specialised palliative home-care teams.

\section{Results}

Patient demographics and tumour characteristics are presented in Table 1 . The mean age of the patients was 67 years, and majority of them were male. Forty-seven (77\%) had major comorbidities of which the most common ones were hypertension in $25(41 \%)$ patients, coronary heart disease in $11(18 \%)$, and diabetes in $10(16 \%)$ patients.

Fourteen (25\%) patients had local or locally advanced disease, $16(26 \%)$ had locally recurrent and $30(49 \%)$ metastatic disease. For 25 (42\%) of the patients, palliative care was the primary treatment without any previous local or systemic disease-specific treatments.

\section{Palliative period}

Median survival after the primary diagnosis was 11 months (range 3 weeks-11.9 years). After referral to the Palliative Center, the median survival was 3 months (range 0-16) (Fig. 1). Thirty-four (56\%) of the patients were referred to the Palliative Center by the multi-disciplinary Head and Neck tumour board, 12 (20\%) by medical oncologists and six $(10 \%)$ by radiation oncologists. The remaining patients were referred by other specialist units. 
Table 1 Patient characteristics $(n=60)$

Age years (range)

$67(28-88)$

Gender

Male

$49(82 \%)$

Female

$11(18 \%)$

Place of living during the palliative phase

Home

$51(85 \%)$

Sheltered home

Hospital ward

$1(2 \%)$

Other

$3(5 \%)$

Marital status

Cohabitant

$30(49 \%)$

Single

$29(48 \%)$

Missing data

Major comorbidities

$1(2 \%)$

$47(78 \%)$

Tumour site

Oropharynx

Oral cavity

Salivary gland

Nasopharynx

Hypopharynx

Paranasal sinuses

Larynx

Histology

Squamous cell carcinoma

Adenoid cystic carcinoma

Melanoma

$20(33 \%)$

$23(38 \%)$

$3(5 \%)$

$2(3 \%)$

$4(7 \%)$

$2(3 \%)$

$6(10 \%)$

$51(85 \%)$

$4(6 \%)$

$1(2 \%)$

$1(2 \%)$

$1(2 \%)$

$2(3 \%)$

Unknown

$1(2 \%)$

$6(10 \%)$

$5(8 \%)$

$38(62 \%)$

$1(2 \%)$

$8(13 \%)$

$1(2 \%)$

Missing

$3(5 \%)$

$21(34 \%)$

$10(17 \%)$

$26(43 \%)$

Palliative treatment

$6(38 \%)$

$2(12 \%)$

Chemotherapy

Treatment for metastatic disease

Chemotherapy

$11(38 \%)$

$19(62 \%)$

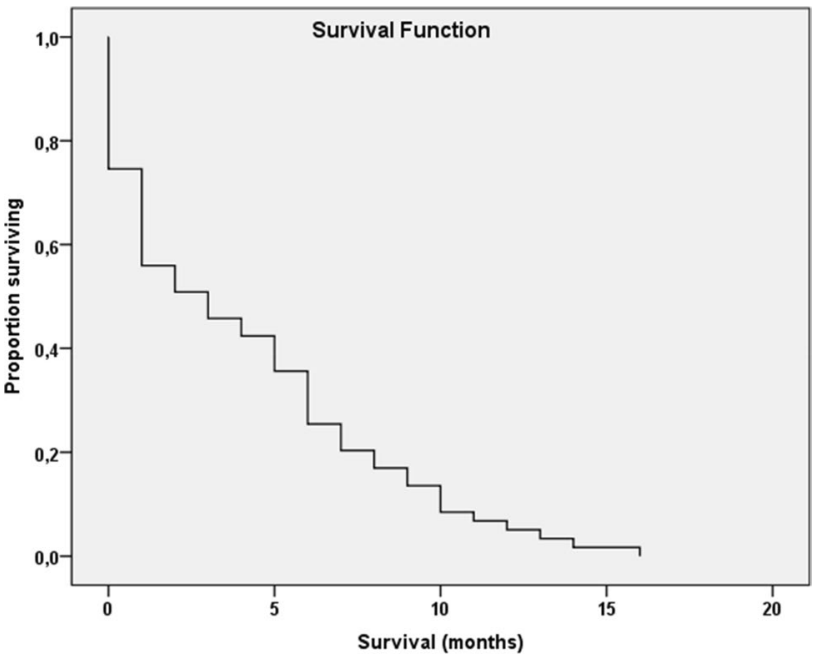

Fig. 1 Overall survival from the beginning of palliative treatment decision

\section{Palliative treatment}

Palliative treatments are described in Table 2.

\section{Radiotherapy and surgery}

None of the patients received palliative surgery during the follow-up period. Twenty-seven (45\%) patients had PEG tube and 17 (28\%) had tracheostomy.

A total of $33(55 \%)$ patients received palliative radiotherapy. Twenty-four (73\%) of these patients received RT

Table 2 Palliative treatment $(n=60)$

\begin{tabular}{lc}
\hline Treatment & $N(\%)$ \\
\hline Palliative RT & $33(55 \%)$ \\
Psychosocial support & $4(7 \%)$ \\
Nutrition counselling & $4(7 \%)$ \\
Physiotherapy & $1(2 \%)$
\end{tabular}

Medication for symptom control

Opioids at any phase of palliative treatment $\quad 59(98 \%)$

Long-acting strong opioids $\quad 42(70 \%)$

Laxatives

$46(77 \%)$

Antiemetics

$26(43 \%)$

Benzodiazepines

$24(40 \%)$

Antidepressants

$11(18 \%)$

$17(28 \%)$

$27(45 \%)$

$23(38 \%)$

$8(13 \%)$

$11(18 \%)$

$4(7 \%)$ 
to the primary area (primary tumour, local recurrence or locoregional lymph node metastases). Of them, six had received prior RT as a part of the primary treatment with a curative intent. Fourteen (42\%) of the 33 patients received palliative RT to distant metastatic sites, such as bone, lungs or thoracic lymph nodes, central nervous system, and subcutaneous or soft tissue metastases.

In the treatment of primary area, conventionally fractionated RT with 30 in 3 Gy daily fractions was used ten times, 20 in 4 Gy daily fractions six times and 15 in 3 Gy daily fractions twice. Three patients received one course of the "quad shot" [17] accelerated palliative RT with 3.5 Gy twice a day for 2 days up to a total dose of 14 Gy. Ten single-fraction courses with either 8 or 6 Gy were given. In addition, despite the palliative intent, two patients received radical RT (one with $66 \mathrm{~Gy}$ and one 70 Gy with concomitant weekly cisplatin, both with 2 Gy daily fractions) to the primary tumour area in an attempt to achieve better local control. The distant lesions were treated with 30 in 3 Gy fractions five times, 20 in 4 Gy fractions eight times, 15 in 3 Gy fractions twice, and with a single fraction three times. The mean number of palliative RT courses per patient was 1.4 (range 1-3). Eleven patients received two or more courses of palliative RT to the same site; seven of the re-irradiations were given to the primary tumour area.

\section{Medical treatment}

The medical treatments are listed in Table 3. Opioid use increased during the palliative phase: at the entry to the Palliative Center $30 \%$ of the patients were using longacting strong opioids and 32\% were using weak opioids. At the last visit to the Palliative Center the corresponding rates were 70 and $8 \%$, respectively. Palliative sedation was performed for one patient.

\section{Complications}

Infection was the most common severe complication during the palliative period ( 24 patients, $40 \%$ ) followed by bleeding in $11(18 \%)$, delirium in seven (12\%), and airway obstructions in five (8\%) patients. In addition, there were $12(20 \%)$ cases of other complications including pulmonary embolism and spinal cord compression. Four patients had a massive bleeding from airways and one patient died due to it. One patient received an emergency tracheostomy.

\section{Palliative care pathway}

The median number of visits per patient to the Palliative Center was three (range 0-11). In addition, there were a median of three contacts per patient to the Palliative Center by phone (range $0-21$ ).

The total number of visits to emergency units and hospitalisations with the most common reasons are presented in Table 4.

Thirty-four (71\%) patients received specialised palliative home care. Twenty-two (65\%) of the 34 patients with and 15 (58\%) of the 26 patients without specialised home care made at least one visit to an emergency department (ED). The total number of the ED visits for these two groups was 35 and 40, respectively, and the mean number of visits per patient was 1 and 1.5 , respectively. The median number of hospitalisations at a university hospital for these two groups was 0.4 (range $0-3$ ) and 0.6 (range 0-4), respectively.

\section{Place of death}

Fifty-nine (98\%) out of the 60 patients died during the follow-up period. Fifteen patients $(25 \%)$ died at home, nine patients (15\%) died in a hospice, $14(23 \%)$ at a specialised end-of-life care ward in a community hospital, $16(27 \%)$ in a general primary care hospital ward, three $(5 \%)$ at the university hospital and three (5\%) in a nursing home. Twelve (35\%) out of the 34 patients who were supported by a specialised

Table 3 Medical treatments

\begin{tabular}{|c|c|c|c|c|c|c|}
\hline & \multicolumn{3}{|c|}{ Entry to the Palliative Centre } & \multicolumn{3}{|c|}{ Last visit to the Palliative Centre } \\
\hline & $n$ & Mean dose & Range & $n$ & Mean dose & Range \\
\hline Strong opioids & 18 & & & 42 & & \\
\hline Oxycodone & 9 & $54 \mathrm{mg} /$ day & $10-280 \mathrm{mg} /$ day & 16 & 90 mg/day & $10-360 \mathrm{mg} /$ day \\
\hline Fentanyl (transdermal) & 9 & $72 \mu / \mathrm{h}$ & $12-300 \mu / \mathrm{h}$ & 22 & $68 \mu / \mathrm{h}$ & $12-300 \mu / \mathrm{h}$ \\
\hline Morphine & 0 & & & 4 & 49 mg/day & 4-180 mg/day \\
\hline Weak opioids & 19 & & & 5 & & \\
\hline Codeine & 11 & $38 \mathrm{mg} /$ day & $30-120 \mathrm{mg} /$ day & 4 & $30 \mathrm{mg} /$ day & $30 \mathrm{mg} /$ day \\
\hline Tramadol & 7 & $150 \mathrm{mg} /$ day & $50-500 \mathrm{mg} /$ day & 0 & & \\
\hline Buprenorphine (transdermal) & 1 & $20 \mu / \mathrm{h}$ & $20 \mu / \mathrm{h}$ & 1 & $5 \mu / \mathrm{h}$ & $5 \mu / \mathrm{h}$ \\
\hline Short-acting opioids & 18 & & & 51 & & \\
\hline
\end{tabular}


Table 4 Use of various resources during the palliative period $(n=60)$

\begin{tabular}{|c|c|}
\hline Number of emergency visits & $N(\%)$ \\
\hline 0 & $24(40 \%)$ \\
\hline 1 & $18(30 \%)$ \\
\hline 2 & $8(13 \%)$ \\
\hline 3 & $5(8 \%)$ \\
\hline 4 & $1(2 \%)$ \\
\hline 5 & $2(3 \%)$ \\
\hline 6 & $2(3 \%)$ \\
\hline \multicolumn{2}{|l|}{ Reason for emergency visit } \\
\hline Infection & $12(22 \%)$ \\
\hline Pain & $11(14 \%)$ \\
\hline Dyspnea & $11(14 \%)$ \\
\hline Coping problem & $8(10 \%)$ \\
\hline Bleeding & $5(6 \%)$ \\
\hline Other & $27(35 \%)$ \\
\hline \multicolumn{2}{|c|}{ Number of hospitalisations at university hospital } \\
\hline 0 & $40(66 \%)$ \\
\hline 1 & $13(21 \%)$ \\
\hline 2 & $6(10 \%)$ \\
\hline 3 & $1(2 \%)$ \\
\hline 4 & $1(2 \%)$ \\
\hline 5 & 0 \\
\hline 6 & 0 \\
\hline \multicolumn{2}{|l|}{ Reason for hospitalisation } \\
\hline End-of-life care & 0 \\
\hline Infection & 9 \\
\hline Coping problem & 4 \\
\hline Pain & 3 \\
\hline Delirium & 1 \\
\hline Bleeding & 2 \\
\hline Other & 10 \\
\hline
\end{tabular}

home care team, and three (12\%) out of the 26 patients without specialised home care died at home. There was no difference in the number of deaths at home between single-living and cohabitant patients.

\section{Discussion}

End-stage HNCs are characterised by heavy symptom burden and short life expectancy. The symptoms related to locoregional tumour growth impact essential human functions such as breathing, speaking, eating and swallowing. Facial mutilation as a result of tumour growth, local treatment or both can cause major functional disability and have a negative impact on the integrity of body image [18]. Difficult physical symptoms and disfigurement cause enormous psychological distress not only to patients but also to their families and caregivers [19]. This emphasises the importance of a specialised, well-organised palliative-care pathway.

Majority of HNC patients are diagnosed at an advanced stage when treatment options are limited and thus, their prognosis in general remains poor. In the present study, three quarters $(77 \%)$ of patients had a locally advanced or metastatic disease. More than every third patient (42\%) was referred to the Palliative Center at first presentation of the disease; this represents $9 \%$ of the newly diagnosed patients during that year in this university hospital region [1]. This proportion is comparable to the previous nationwide studies performed in Finland [2-5].

Overall survival in the present cohort was relatively short with a median of 11 months after primary diagnosis and only 3 months after the decision of withholding cancer-specific treatments. Previous retrospective studies [6, 7] have reported similarly short survival rates, with certain factors, such as disease stage, primary tumour location, palliative interventions, patient performance status, and comorbidity, having an impact on the prognosis. In their study of 808 HNC patients without cancer specific treatments, Kowalski et al. [6] reported a 3.8 months median overall survival, whereas Ledeboer et al. [7] reported that the median length of the palliative period was 5.3 months.

Over half the patients received palliative RT, and onethird of them went through at least two courses of RT during follow-up. Palliative RT was given in most cases to the head and neck area; either due to locoregional recurrence after curative-intended primary treatment, or symptomatic locoregional tumour growth in a primarily incurable disease. The most common RT dose to the primary area was 30 with $3 \mathrm{~Gy}$ daily fractions. In addition to locoregional radiotherapy, a quarter of the patients received palliative radiotherapy to various distant lesions, most of which were uncomplicated bone metastases or metastases in the thoracic area. These were typically treated with single fractions or short fractionated courses.

Although there is evidence that locoregional palliative treatments are beneficial for patients with non-curable locally advanced HNC [20], there is still no clear consensus on the optimal scheduling of palliative RT for these patients. Short hypofractionated courses of palliative RT are supported by several prospective [17, 21, 22] and retrospective [23-26] studies demonstrating both good tolerability and symptom control in this population with a very limited life expectancy. The high number of palliative locoregional reirradiations in our patient cohort emphasises the need for further investigations to determine the optimal palliative RT protocol for these patients.

There were a limited number of severe local complications among this highly symptomatic patient group, suggesting that elective procedures to avoid major complications were successfully used. Likewise, locoregional 
complications were rarely the reason for ED visits or hospitalisations. Percutaneous nutrition and prophylactic tracheostomy were widely used among our patients, and only one patient needed an emergency tracheostomy. The use of strong opioids was common, and the number of opioid users, as well as the opioid doses, increased significantly during the follow-up.

However, although most patients had a round-the-clock access to specialised palliative care services, a relatively large proportion of the patients did visit an ED (66\%) or got hospitalised in a university hospital (35\%) during the last months of their lives. The leading causes of ED visits and hospitalisations were infections, pain, dyspnoea, and coping difficulties. For comparison, in a small retrospective study on 32 patients with HNC [8], bleeding was the most common reason for visiting an emergency unit, followed by pain, respiratory symptoms and swallowing problems. Some of the emergency visits and hospitalisations in our patient cohort would likely have been avoidable. This further emphasises the need for more intense follow-up of these patients.

According to a European study [27], $60 \%$ of people would prefer dying at home. A recent Cochrane review [28] reported that home-based end-of-life care services increase the likelihood of home deaths. In accordance with the Cochrane review, also in our cohort an access to specialised palliative home care seemed to increase the home-death rates (35\% of the patients with specialised home care vs. $11 \%$ without it), but still, even with the support of specialised palliative home care, only one-third of the deaths in our cohort did occur at home. These low home-death rates could partly be explained by the significant symptom burden, high rate of infections and the fact that half the patients were living alone, even though no difference in the rate of home deaths was found between the single-living and cohabitant patients. Further, the patients with a specialised palliative home care have a direct access to community hospital palliative wards whenever needed. Since some of the difficult symptoms may be easier to manage at a hospital ward, this could have further reduced the home-death rates in this cohort.

There were some limitations in this study that should be pointed out. First, this was a small retrospective study, which limits further statistical analysis and makes this report a descriptive one. Second, the symptoms and their development were not systematically reported in the patient records. Strength of this study is its population-based nature as all palliative-phase HNC patients in this university hospital region during one year were included. This area represents almost one-third of the Finnish population and there are no reasons to expect that the present series would differ from those in other regions in Finland.

In conclusion, a systematic collaboration with a Palliative Care Unit seems to assure sufficient end-of-life care to HNC patients with non-curable disease. Specialised palliative care pathway, particularly home care, increases the possibility for end-of-life care at home. Due to heavy symptom burden and short life expectancy in this patient population it is obvious that intense palliative care pathway from Cancer Center to home care is needed to ensure good quality of palliative care and a dignified death.

\section{Compliance with ethical standards}

Funding This study was funded by the Helsinki University Hospital Research Fund (Grant number TYH2015204).

Conflict of interest All authors declare no conflict of interest.

Ethical approval All procedures performed in this study involving human participants were in accordance with the ethical standards of the institutional research ethics committee and with the 1964 Helsinki declaration and its later amendments or comparable ethical standards. This is a retrospective chart review and thus, no approval by the Research Ethics Committee was needed according to Finnish Law. An institutional research permission was granted.

Informed consent This is a retrospective chart review and thus, no informed consent was needed according to the Finnish Law (and all patients were dead at the time of the study).

\section{References}

1. http://www.cancerregistry.fi. Accessed 24 Sept 2017

2. Haapaniemi A et al (2016) Laryngeal cancer in Finland: a 5-year follow-up study of 366 patients. Head Neck 38(1):36-43

3. Koivunen P et al (2012) A national series of 244 sinonasal cancers in Finland in 1990-2004. Eur Arch Otorhinolaryngol 269(2):615-21

4. Makitie AA et al (2007) Oral tongue carcinoma and its treatment in Finland. Eur Arch Otorhinolaryngol 264(3):263-267

5. Makitie AA et al (2006) Oropharyngeal carcinoma and its treatment in Finland between 1995-1999: a nationwide study. Eur Arch Otorhinolaryngol 263(2):139-43

6. Kowalski LP, Carvalho AL (2000) Natural history of untreated head and neck cancer. Eur J Cancer 36(8):1032-1037

7. Ledeboer QC et al (2011) Survival of patients with palliative head and neck cancer. Head Neck 33(7):1021-1026

8. Ethunandan M et al (2005) Quality of dying in head and neck cancer patients: a retrospective analysis of potential indicators of care. Oral Surg Oral Med Oral Pathol Oral Radiol Endodol 100(2): 147-52

9. Price KA et al (2009) Symptoms and terminal course of patients who died of head and neck cancer. J Palliat Med 12(2):117-118

10. Lin YL, Lin IC, Liou JC (2011) Symptom patterns of patients with head and neck cancer in a palliative care unit. J Palliat Med 14(5):556-559

11. Weis J (2015) Psychosocial care for cancer patients. Breast care 10(2):84

12. Temel JS et al (2010) Early palliative care for patients with metastatic non-small-cell lung cancer. N Engl J Med 363(8):733-42

13. Bakitas M et al (2009) Effects of a palliative care intervention on clinical outcomes in patients with advanced cancer: the project ENABLE II randomised controlled trial. JAMA 302(7):741-749 
14. Simmermann C et al (2014) Early palliative care for patients with advanced cancer: a cluster-randomised controlled trial. Lancet 383(9930):1721-1730

15. Scibetta $C$ et al (2016) The costs of waiting: implications of the timing of palliative care consultation among a cohort of decedents at a comprehensive cancer center. J Palliat Med 19(1):69-75

16. Blackhall LJ et al (2016) CARE track for advanced cancer: impact and timing of an outpatient palliative care clinic. J Palliat Med 19(1):57-63

17. Corry J et al (2005) The 'QUAD SHOT' - a phase II study of palliative radiotherapy for incurable head and neck cancer. Radiother Oncol 77(2):137-42

18. Wells M et al (2015) Distress, concerns and unmet needs in survivors of head and neck cancer: a cross-sectional survey. Eur J Cancer Care (Engl) 24(5):748-60

19. Balfe $M$ et al (2017) Social networks, social support and social negativity: a qualitative study of head and neck cancer caregivers' experiences. Eur J Cancer Care (Engl) 26(6). https://doi. org/10.1111/ecc.12619. Epub 2016 Dec 22

20. Carvalho AL, Salvajoli JV, Kowalski LP (2000) A comparison of radiotherapy or radiochemotherapy with symptomatic treatment alone in patients with advanced head and neck carcinomas. Eur Arch Otorhinolaryngol 257(3):164-167

21. Mohanti BK et al (2004) Short course palliative radiotherapy of $20 \mathrm{~Gy}$ in 5 fractions for advanced and incurable head and neck cancer: AIIMS study. Radiother Oncol 71(3):275-80
22. Porceddu SV et al (2007) Hypofractionated radiotherapy for the palliation of advanced head and neck cancer in patients unsuitable for curative treatment- "Hypo Trial". Radiother Oncol 85(3):456-62

23. Al-mamgani A et al (2009) Hypofractionated radiotherapy denoted as the "Christie scheme": an effective means of palliating patients with head and neck cancers not suitable for curative treatment. Acta Oncol 48(4):562-70

24. Kancherla KN et al (2011) The role of split-course hypofractionated palliative radiotherapy in head and neck cancer. Clin Oncol (R Coll Radiol) 23(2):141-148

25. Monnier L et al (2013) Hypofractionated palliative radiotherapy for advanced head and neck cancer: the IHF2SQ regimen. Head Neck 35(12): 1683-1688

26. Agarwal JP et al (2008) Hypofractionated, palliative radiotherapy for advanced head and neck cancer. Radiother Oncol 89(1):51-6

27. http://www.eapcnet.eu/Themes/Organisation/DevelopmentinEurope/EAPCAtlas2013.aspx. Accessed 24 Sept 2017

28. Shepperd S, Gonçalves-Bradley D, Straus SE, Wee B (2016) Hospital at home: home-based end-of-life care. Cochrane Database Syst Rev. https://doi.org/10.1002/14651858.CD009231.pub2 